\title{
Precluding Pregnant Women from COVID-19 Vaccine Trials is Cause for Concern
}

Michelle Winokur, DrPH, and the AfPA Governmental Affairs Team, Alliance for Patient Access (AfPA)

The Alliance for Patient Access (allianceforpatientaccess.org), founded in 2006, is a national network of physicians dedicated to ensuring patient access to approved therapies and appropriate clinical care. AfPA accomplishes this mission by recruiting, training and mobilizing policy-minded physicians to be effective advocates for patient access. AfPA is organized as a non-profit 501(c)(4) corporation and headed by an independent board of directors. Its physician leadership is supported by policy advocacy management and public affairs consultants. In 2012, AfPA established the Institute for Patient Access (IfPA), a related 501(c) (3) non-profit corporation. In keeping with its mission to promote a better understanding of the benefits of the physician-patient relationship in the provision of quality healthcare, IfPA sponsors policy research and educational programming.

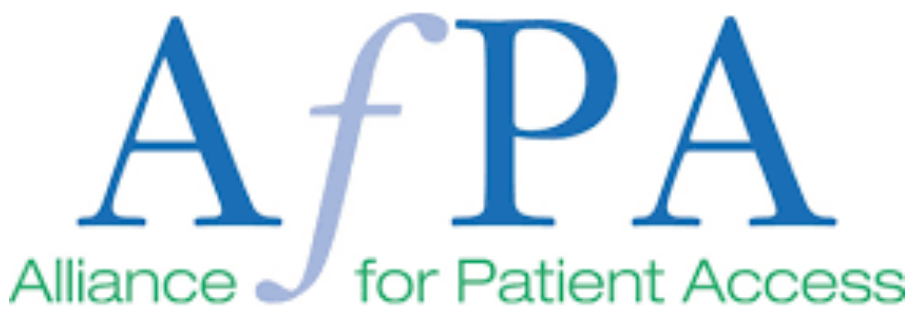

It is normal to test vaccines in healthy adults first. They have the lowest risk should a side effect occur. It is the same reason older adults, young children, and people with certain health conditions are typically not included in early trials. However, people in each of these groups have already been included in COVID-19 vaccine trials, while one group is notably missing: pregnant women. (1)

\section{"However, people in each of these groups have already been included in COVID-19 vaccine trials, while one group is notably missing: pregnant women. (1)"}

Researches would do well to find a way to safely include pregnant women - and soon. Having a vaccine that hundreds of millions of people worldwide cannot take should be concerning.

Women of reproductive age comprise a sizable portion of the workforce, and they are overrepresented in many professions that are less likely to be able to work remotely. They are first responders, health and child care providers, and educators, among other front-line jobs. Vaccinating pregnant women will not only protect them from serious COVID-19 complications, but it also will benefit their communities and those they serve.

The Food and Drug Administration recognized the necessity of testing vaccines in pregnant women. In June, the agency issued guidance that called upon vaccine developers to include not just diverse populations, elderly individuals and those with medical comorbidities, but also to "provide data to support [vaccine] use during pregnancy."(2)

From influenza to the zika virus to Ebola, pregnant women experience more severe health outcomes, up to and including maternal death and fetal loss.(3) Data released earlier this month from the Centers for Disease Control and Prevention show similar trends are likely for pregnant women with confirmed SARS-CoV-2 infection.

$$
\begin{aligned}
& \text { "The surveillance data representing the } \\
& \text { experience of more than } 20,000 \text { pregnant } \\
& \text { women suggest they are more likely } \\
& \text { to be hospitalized due to COVID-19- } \\
& \text { related complications than non-pregnant } \\
& \text { women.(4)" }
\end{aligned}
$$

The surveillance data representing the experience of more than 20,000 pregnant women suggest they are more likely to be hospitalized due to COVID-19-related complications than non-pregnant women.(4) Additionally, pregnant women appear to be at increased risk of needing intensive care-level treatment and ventilator support.

While the more recent data seem to validate early trends that show pregnant woman are not more likely to die from COVID-19, just knowing they are more vulnerable to severe COVID-19 should be reason enough to work toward an effective vaccine for them. (5)

Additionally, vaccinating pregnant women against COVID-19 could benefit their unborn babies. Research has proven that pregnant women who are vaccinated against certain diseases, flu, and whooping cough, for example, pass along antibodies to their babies. (6) These antibodies give babies some immunity until they are old enough to be vaccinated themselves. The question as to whether or not unborn babies are similarly protected from COIVD-19 will remain until pregnant women are included in clinical trials.

As the months toward the January 2021 target date for delivering a safe, effective COVID-19 vaccine wind down, those involved in the effort should pause to reflect on the effect of their omission, then retool their approach to include pregnant women in COV- 
ID-19 testing.

\section{References}

1. H Branwell. Will Covid-19 vaccines be safe for children and pregnant women? The data, so far, are lacking. Stat. August 19, 2020. https://www. statnews.com/2020/08/19/ will-covid-19-vaccines-be-safe-for-children-and-pregnantwomen-the-data-so-far-are-lackingl

2. FDA News Release: Coronavirus (COVID-19) Update: FDA Takes Action to Help Facilitate Timely Development of Safe, Effective COVID-19 Vaccines. June 30, 2020. https:// www.fda.gov/news-events/press-announcements/coronavirus-covid-19-update-fda-takes-action-help-facilitate-timelydevelopment-safe-effective-covid

3. Pregnancy Research Ethics for Vaccines, Epidemics, and New Technologies (PREVENT). http://vax.pregnancyethics. orgl

4. $\quad$ Centers for Disease Control and Prevention. Data on COVID-19 during Pregnancy. September 3, 2020. https://www. cdc.gov/coronavirus/2019-ncov/cases-updates/specialpopulations/pregnancy-data-on-covid-19.html

5. S Ellington, $P$ Strid et al. Characteristics of Women of Reproductive Age with Laboratory-Confirmed SARS-CoV-2 Infection by Pregnancy Status - United States, January 22-June 7, 2020. MMWR Morb Mortal Wkly Rep. 2020 Jun 26; 69(25): 769-775. Published online 2020 Jun 26. doi: 10.15585/mmwr.mm6925a1

6. Health and Human Services. Vaccines for Pregnant Women. https://www. vaccines.gov/who and when/ pregnant\#: :text=Vaccines\%20can\%20help\%20protect $\% 20$ both, called\%20antibodies\%20to\%20their\%20babies.

The author has not indicated any disclosures.

NT

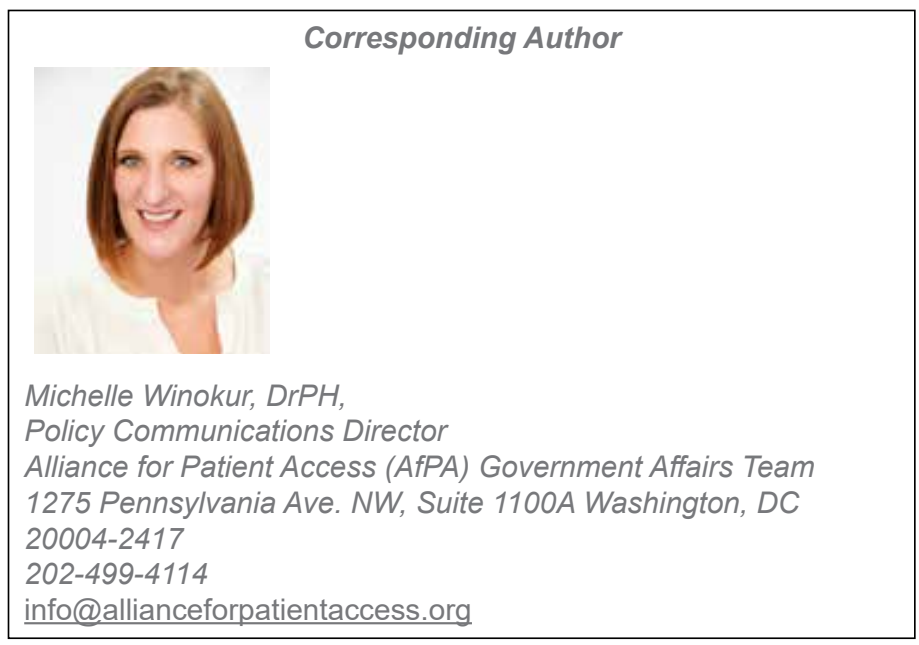

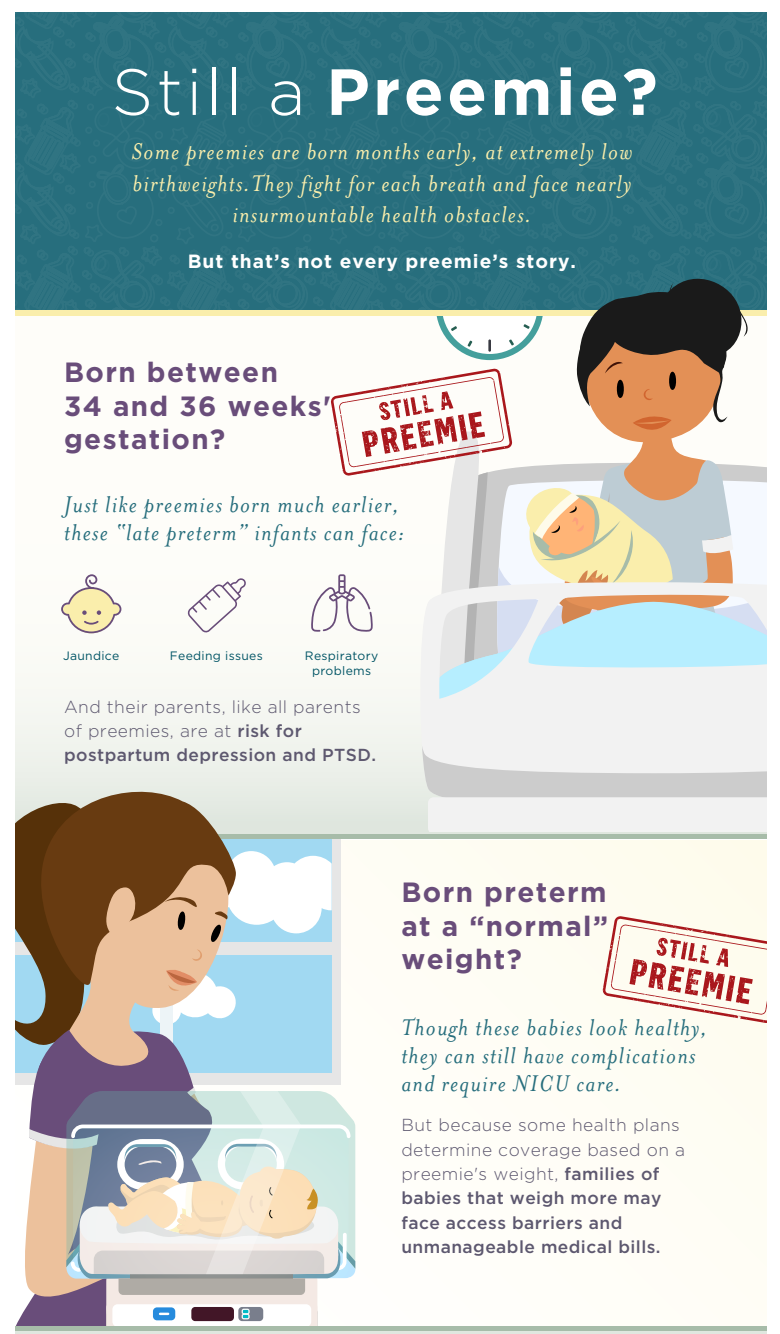

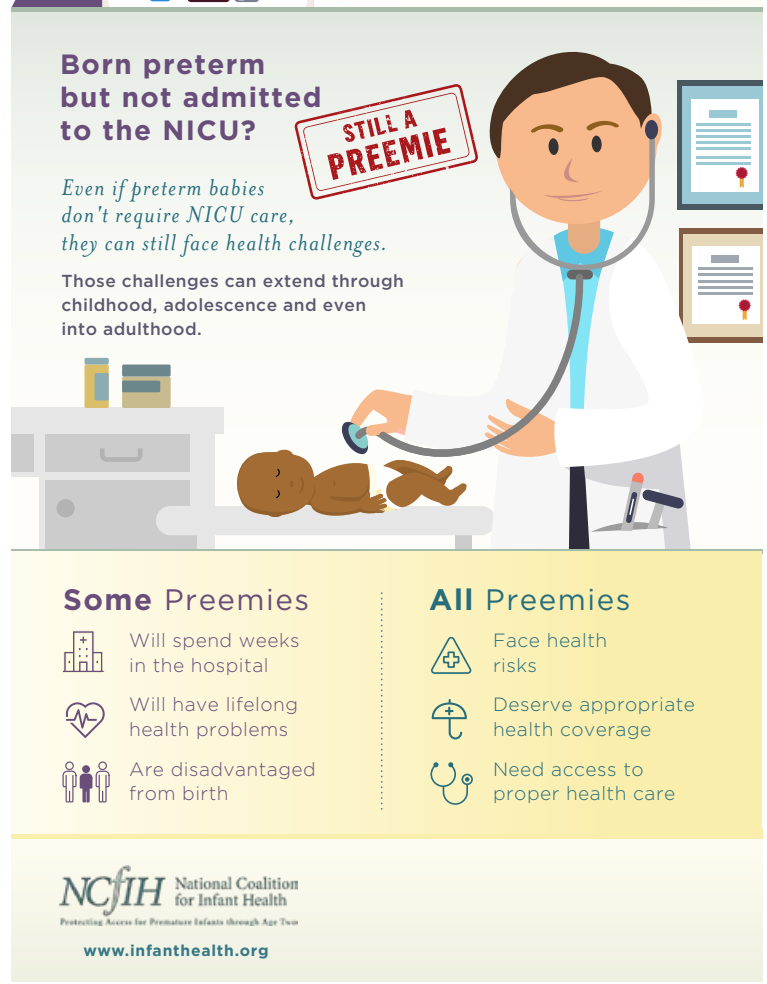

